\title{
Adaptabilidade de Carreira e Empregabilidade na Transição Universidade-Trabalho: Mediação das Respostas Adaptativas
}

\author{
Mariana Rita Machado Ladeira ${ }^{1}$ \\ Marina Cardoso de Oliveira ${ }^{T}$ \\ Lucy Leal Melo-Silva ${ }^{2}$ \\ Maria do Céu Taveira ${ }^{3}$ \\ ${ }^{1}$ Universidade Federal do Triângulo Mineiro, Uberaba, $M G$ \\ ${ }^{2}$ Universidade de São Paulo, Ribeirão Preto, SP \\ ${ }^{3}$ Universidade do Minho, Braga, Portugal
}

\begin{abstract}
Resumo
O objetivo desse estudo foi analisar o poder preditivo da adaptabilidade de carreira na empregabilidade percebida, considerando o efeito mediador das respostas adaptativas nessa relação. Participaram da pesquisa 387 estudantes universitários. Para a coleta de dados foram utilizadas a Escala de Adaptabilidade de Carreira, a Escala de Desenvolvimento de Carreira de Estudantes Universitários e a Escala de Empregabilidade. Os resultados ressaltaram o poder preditivo da adaptabilidade de carreira na empregabilidade percebida e um efeito mediador parcial das respostas adaptativas nessa relação. A preocupação e identidade de carreira foram as dimensões da adaptabilidade e das respostas adaptativas mais significativas para predizer a empregabilidade. Por fim, são discutidas as implicações dos resultados na literatura vocacional, enfatizando a transição universidade-trabalho.

Palavras-chave: adaptabilidade de carreira; empregabilidade; transição de carreira
\end{abstract}

\section{Career Adaptability and Employability in the University-Work Transition:} Mediating Adaptive Responses

\begin{abstract}
The objective of this study was to analyze the relationships established between career adaptability and perceived employability, considering the mediating effect of the adaptive responses in these relationships, in a sample of 387 university students. For data collection, we used the Career Adaptability Scale, the University Student Career Development Scale, and the Employability Scale. The results suggest positive and moderate associations between the constructs studied, besides a possible partial mediating effect of the adaptive responses in relation to the perceived employability. Additionally, the results indicate the prediction of career adaptability and adaptive responses in relation to perceived employability, especially in the career dimension and career identity. Finally, we discuss the implications of the results in the vocational literature, emphasizing the university-work transition. Keywords: career adaptability; employability; career transition
\end{abstract}

\section{Adaptabilidad Profesional y Empleabilidad en la Transición Universidad-Trabajo: Mediación de Respuestas Adaptativas}

\section{Resumen}

El objetivo de este estudio fue analizar el poder predictivo de la adaptabilidad profesional en la empleabilidad percibida, considerando el efecto mediador de las respuestas adaptativas en esa relación, participando de esta investigación 387 estudiantes universitarios. Para la recolección de datos fueron utilizadas: Escala de Adaptabilidad Profesional, Escala de Desarrollo Profesional de Estudiantes Universitarios y Escala de Empleabilidad. Los resultados señalaron el poder predictivo de la adaptabilidad profesional en la empleabilidad percibida, además de un efecto mediador parcial de las respuestas adaptativas en esa relación, cumpliéndose de esta forma el objetivo propuesto. La preocupación e identidad profesional, fueron las dimensiones de adaptabilidad y de las respuestas adaptativas más significativas para predecir la empleabilidad. Por último, se discuten las implicaciones de los resultados en la literatura vocacional, enfatizando la transición universidad-trabajo.

Palabras clave: adaptabilidad profesional; empleabilidad; transición profesional

\section{Introdução}

O mundo do trabalho contemporâneo tem sido caracterizado pela instabilidade e dinamicidade nas relações de trabalho e nas trajetórias de carreira (Guilbert, Bernaud, Gouvernet, \& Rossier, 2015). Em resposta às inúmeras e velozes transformações, a concepção de carreira foi ampliada sendo construída a partir do conjunto de decisões tomadas pelo trabalhador, 
no contexto e lógica de sua trajetória de vida, não se restringindo a uma sequência linear de empregos ou ocupações (Savickas et al., 2009).

Assim, a carreira passa a ser compreendida como uma construção social, formada pelas significações de memórias passadas, experiências atuais, aspirações e expectativas futuras relacionadas ao trabalho, emergindo, portanto, de um processo ativo (Savickas, 2013). Nesse sentido, os sujeitos vão construindo a sua carreira à medida que se relacionam e tomam decisões, com o objetivo de se sentirem satisfeitos e atuantes na sociedade (Savickas, 2005).

Deste modo, para construir a carreira nestas primeiras décadas do século XXI, os trabalhadores precisarão desenvolver um conjunto de competências que os ajudem a enfrentar e se adaptar às exigências do mundo do trabalho cada vez dinâmico e sem fronteiras, demarcado por transições cada vez mais frequentes e múltiplas trajetórias de carreiras (Savickas, 2009). Buscando compreender as variáveis que afetam a construção e o sucesso na carreira na atualidade, pesquisas recentes têm ressaltado o poder preditivo e mediador da adaptabilidade de carreira (Carvalho, Moreira \& Ambiel, 2017).

A adaptabilidade de carreira é um conceito central na teoria de construção da carreira e, mais amplamente, no campo da Psicologia Vocacional contemporânea (Brown \& Lent, 2016). De modo geral, a adaptabilidade de carreira denota a prontidão e os recursos de um indivíduo para lidar com tarefas atuais e iminentes de desenvolvimento de carreira, bem como as transições ocupacionais e os traumas pessoais (Savickas, 2013). Desenvolve-se por meio da interação entre o mundo interno e externo, por isso, a cultura e as condições do contexto influenciam significativamente o seu aprimoramento (Savickas \& Porfeli, 2012).

Nesse sentido, McMahon, Watson e Bimrose (2012) delimitaram três características importantes no âmbito da adaptabilidade de carreira: (a) depende do contexto; (b) manifesta-se de maneiras diferentes em diferentes indivíduos; (c) está presente nas diversas fases de desenvolvimento da carreira e não apenas nos momentos de transição. Do ponto de vista teórico, existe consenso de que a adaptabilidade de carreira é um recurso psicossocial fundamental para enfrentar os desafios contemporâneos para a construção da carreira $\mathrm{e}$, por isso, tem despertado o interesse dos pesquisadores, sendo este conceito um dos mais estudados na atualidade, tornando-se objeto de investigação em diferentes partes do mundo como apontam os estudos de
Savickas e Porfeli (2012); Hirschi, Herrmann e Keller (2015); e Rudolph, Lavigne e Zacher (2017). No contexto brasileiro destacam-se os estudos de Teixeira, Bardagi, Lassance, Magalhães e Duarte (2012), Audibert e Teixeira (2015); Ambiel, Carvalho, Martins e Tofoli (2016); Ambiel, Hernández e Martins (2016); e Carvalho, Moreira e Ambiel (2017).

Nos dias de hoje, as pesquisas sobre a adaptabilidade de carreira têm sido enquadradas dentro de um modelo mais amplo denominado na literatura como Modelo da Adaptação de Carreira ou Career Construction Model of Adaptation (Hirschi et al., 2015; Rudolph et. al., 2017; Savickas \& Porfeli, 2012; Savickas, 2013). Nesse modelo, esquematizado na Figura 1, a adaptação seguiria uma sequência que vai desde a prontidão adaptativa (adaptivity) passando pelos recursos de adaptabilidade (adaptability), que desencadeariam as respostas adaptativas (adapting) que, por sua vez, promoveriam os resultados de adaptação (adaptation) (Hirschi, et al., 2015; Rudolph et al., 2017; Savickas \& Porfeli, 2012; Savickas; 2013).

É importante ressaltar que este modelo parte do princípio que a prontidão adaptativa (traços psicológicos), por si só, é insuficiente para dar suporte às respostas adaptativas (crenças e comportamentos de carreira), sendo necessários recursos autorreguladores de adaptabilidade de carreira para lidar com situações em mudança. É pela integração destes diferentes elementos que se alcançam os resultados da adaptação (Savickas \& Porfeli, 2012).

Hirschi et al. (2015) discutem como os conceitos de prontidão adaptativa, adaptabilidade de carreira, respostas adaptativas e resultados da adaptação estão sendo utilizados nas pesquisas quando associados à adaptabilidade de carreira. Em síntese, a prontidão adaptativa, relacionada aos traços de personalidade, promoveria uma maior adaptabilidade de carreira que, por sua vez, desencadearia um conjunto de respostas adaptativas, tais como o planejamento, a decisão, a exploração e a autoeficácia, que levariam ao alcance de bons resultados decorrentes da adaptação ao trabalho e à carreira (Hirschi et al., 2015; Rudolph et al., 2017). Esses construtos são relevantes para a compreensão do conceito empregabilidade, descrito a seguir.

\section{Empregabilidade}

Dentre os diferentes resultados da adaptação de carreira, a empregabilidade percebida tem sido utilizada como variável de resultado em vários estudos (Gamboa, Paixão \& Palma, 2014; Rothwell, Jewell \& Hardie, 


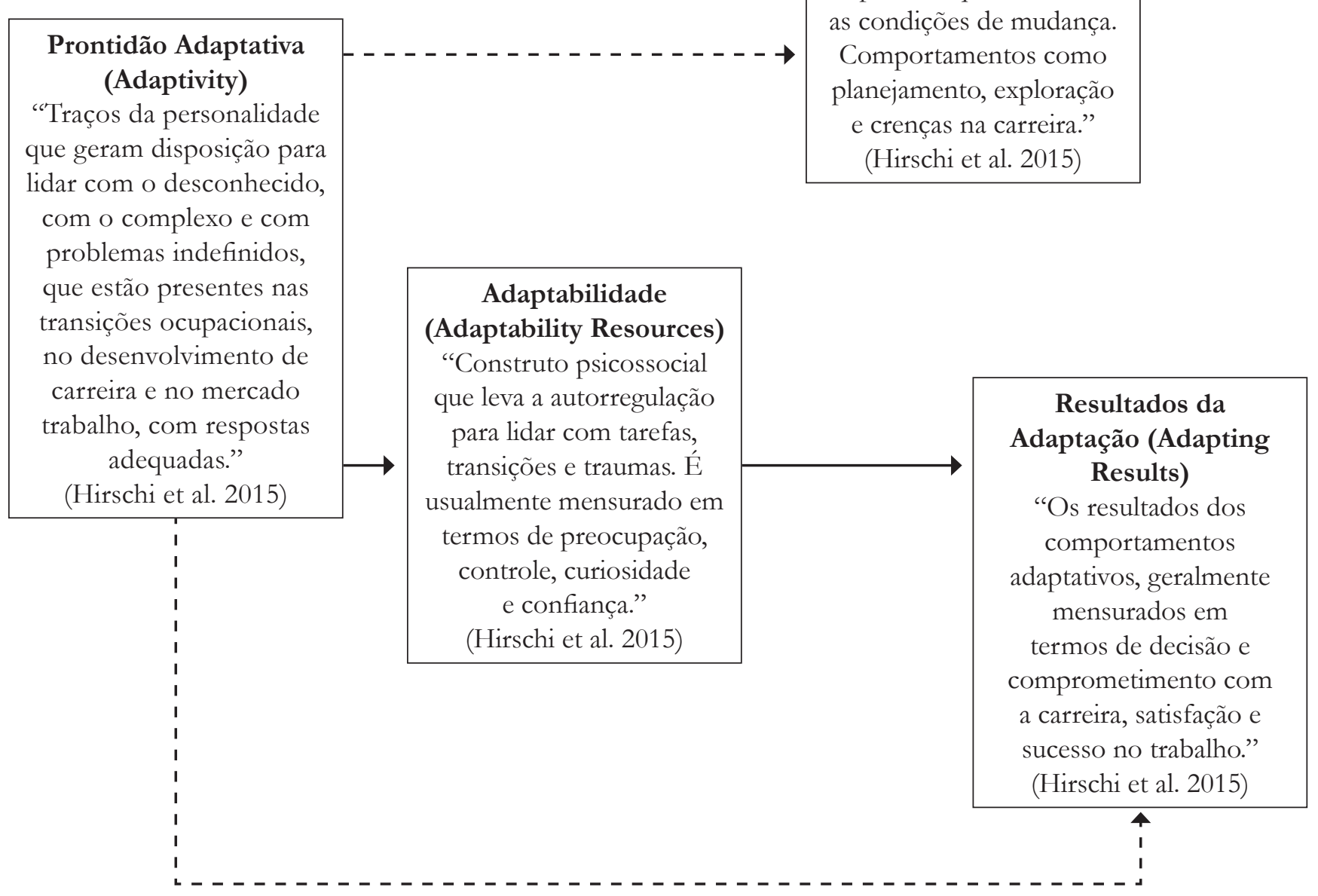

Figura 1. Fragmento conceitual baseado no modelo da Adaptação de Carreira (Rudolph et al., 2017; Savickas, 2005, 2013; Savickas \& Porfeli, 2012; Savickas et al., 2009). Nota: Seta sólida representam relações parcialmente examinadas. As setas tracejadas representam relações não examinadas.

2009; Rudolph et al. 2017). Isso porque, nos últimos anos, devido às transformações na natureza dos contratos de trabalho, a antiga estabilidade no emprego tem sido substituída pela empregabilidade. Nesse sentido, espera-se que os profissionais busquem conhecimentos, aptidões, habilidades e outras características que são valorizadas pelo mercado, como estratégia para manter-se inseridos no mundo do trabalho (Fugate \& Kinicki, 2008).

Lo Presti e Pluviano (2016) definem a empregabilidade como um recurso pessoal que os indivíduos desenvolvem durante sua vida no trabalho e que pode ampliar as chances de sucesso na carreira. A literatura sugere que este conceito é um constructo

\section{Respostas Adaptativas (Adapting Responses)}

"Realizar comportamentos adaptativos que direcionam as condições de mudança. Comportamentos como planejamento, exploração crenças na carreira." (Hirschi et al. 2015) 
relevante, tendo sido observados resultados significativos em vários estudos (Gamboa et al., 2014; McArdle, Waters, Briscoe \& Hall, 2007; Rudolph et al., 2017).

Partindo destas evidências e buscando contribuir com os estudos empíricos sobre Modelo da Adaptação da Carreira, este estudo assume a hipótese de que as respostas adaptativas mediam a relação entre a adaptabilidade de carreira e a empregabilidade percebida. Nesse sentido, o objetivo desse estudo foi analisar o poder preditivo da adaptabilidade de carreira na empregabilidade percebida, considerando o efeito mediador das respostas adaptativas nessa relação.

\section{Método}

\section{Participantes}

Participaram do estudo 387 estudantes universitários regularmente matriculados nos dois últimos semestres da graduação (último ano), predominantemente do sudeste do Brasil ( $\mathrm{n}=368-95,1 \%$ ) e de universidades particulares ( $\mathrm{n}=207-53,5 \%)$. Os participantes foram majoritariamente mulheres $(\mathrm{n}=235$ - 60,7\%), com idades distribuídas entre 18 e 56 anos $(M=24,4-\mathrm{DP}=5,14)$. A maior parte dos participantes cursava sua primeira formação ( $n=365-94,3 \%$ ), tinha pretensão de atuar na área de formação $(\mathrm{N}=371$ $95,9 \%$ e tinha expectativa de concluir a graduação em até 12 meses ( $\mathrm{n}=208-53,7 \%$ ).

Em relação à profissão escolhida, os sujeitos estavam distribuídos entre 38 cursos agrupados em seis grandes áreas, sendo elas: Ciências Exatas e da Terra $(n=17-4,4 \%)$, Engenharias $(n=92-23,8 \%)$, Ciências da Saúde ( $\mathrm{n}=122-31,5 \%)$, Ciências Sociais ( $\mathrm{n}=54$ $14 \%)$, Ciências Humanas ( $\mathrm{n}=86-22,2 \%$ ) e Outros ( $n=16-4,1 \%)$. O curso que obteve o maior número de respondentes foi o de Psicologia ( $\mathrm{n}=77-19,9 \%$ ), seguido pelos cursos de Medicina ( $\mathrm{n}=46-11,6 \%)$ e Engenharia Civil $(\mathrm{n}=43-11,1 \%)$.

\section{Instrumentos}

Para medir a adaptabilidade de carreira foi utilizada a Escala de Adaptabilidade de Carreira (Career Adapt-Abilities) - versão adaptada para o Brasil (Audibert \& Teixeira, 2015; Teixeira, Bardagi, Lassance, Magalhães, \& Duarte, 2012). A adaptabilidade de carreira foi avaliada mediante a resposta do quanto a pessoa acredita ter desenvolvido cada habilidade, numa escala Likert que varia de "muito pouco" (1) a "plenamente" (5). A escala é composta por 24 itens, que por meio de análise fatorial confirmatória agruparam-se em quatro fatores que carregam seis itens cada, quais sejam, Preocupação (Concern), Controle (Control), Curiosidade (Curiosity) e Confiança (Confidence). Para essa amostra foi testado o ajustamento da medida por meio da análise fatorial confirmatória que mostrou índices satisfatórios $\left(\mathrm{X}^{2} /(242)=657,248 ; \mathrm{p}<0,001 ; \mathrm{N}=387 ; \mathrm{X}^{2} / \mathrm{df}=2,716\right.$; $\mathrm{CFI}=0,926$; RMSEA $=0,067 ; \alpha=0,95)$.

As respostas adaptativas foram avaliadas a partir da Escala de Desenvolvimento de Carreira de Estudantes Universitários (Teixeira, Oliveira, Melo-Silva \& Taveira, 2019, submetido à publicação). Este instrumento foi escolhido, pois avalia um conjunto de comportamentos e crenças relacionados às respostas adaptativas. Trata-se de uma medida de autorrelato que avalia quatro dimensões associadas às respostas adaptativas de estudantes universitários, sendo estas: identidade com a carreira, autoeficácia profissional, decisão de carreira, e exploração ampliada de carreira. Para a chave de respostas, foi utilizada uma escala do tipo Likert de cinco pontos $(1=$ "a frase é totalmente falsa a seu respeito" a $5=$ "a frase é totalmente verdadeira a seu respeito"). Para essa amostra foi testado o ajustamento do modelo por meio da análise fatorial confirmatória que mostrou bons índices de ajustamento da medida $\left(\mathrm{X}^{2} /(289)=748,342 ; \mathrm{p}<0,001 ; \mathrm{N}=387 ; \mathrm{X}^{2} /\right.$ $\mathrm{df}=2,589 ; \mathrm{CFI}=0,901 ; \mathrm{RMSEA}=0,064 ; \alpha=0,91)$.

A empregabilidade foi avaliada por meio da Escala de Empregabilidade Percebida (Rothwell, Herbert, \& Rothwell, 2008), validada para a população portuguesa por Gamboa, Paixão e Rothwell (em preparação) citado por Gamboa et al. (2014). Esta escala oferece uma medida interna e externa de empregabilidade. É composta por 16 itens, relacionados ao envolvimento com os estudos, valor da universidade, valor da universidade em um determinado domínio de formação, estatuto de um determinado domínio de formação, procura do mercado de trabalho, procura geral, atenção às oportunidades de trabalho e confiança nas competências. Para os efeitos deste estudo, a Escala de Empregabilidade Percebida foi adaptada para o português do Brasil, a partir de uma análise do português-brasileiro da versão original portuguesa, item a item não tendo sido necessários ajustes semânticos nos itens, pois já estavam adequados semanticamente ao contexto brasileiro. Em seguida procedeu-se um estudo fatorial confirmatório e a análise da fiabilidade. Para essa amostra o ajustamento da medida por meio da análise fatorial confirmatória mostrou índices de ajustamento aceitáveis $\left(X^{2} /(96)=313,330 ; \quad p<0,001 ; \quad N=387 ; \quad X^{2} / d f=3,264\right.$; $\mathrm{CFI}=0,874$; RMSEA $=0,077 ; \alpha=0,82$ ).

Psico-USF, Bragança Paulista, v. 24, n. 3, p. 583-595, jul./set. 2019 


\section{Procedimentos}

Para realização deste estudo, o mesmo foi submetido e aprovado pelo Comitê de Ética em Pesquisa da Universidade das duas primeiras autoras. Os participantes foram contatados por conveniência, e a coleta de dados ocorreu pessoalmente ou via meios eletrônicos: e-mail e facebook. Embora o formato lápis-e-papel e o online sejam diferentes na aparência e na forma de recrutamento e envolvimento dos participantes, há evidências de que os resultados de pesquisas com dados obtidos com esses dois métodos tendem a ser equivalentes (Birnbaum, 2004; Gosling, Vazire, Srivastava, \& John, 2004). Para a aplicação presencial, os participantes responderam o caderno de pesquisa impresso e assinaram o termo de consentimento livre e esclarecido (TCLE). Já os participantes que colaboraram de forma eletrônica, responderam ao caderno de pesquisa e ao TCLE a partir de uma planilha online. Os participantes foram informados acerca da confidencialidade dos dados, assim como do caráter voluntário da sua participação.

\section{Análise dos Dados}

A análise dos dados foi feita a partir do software IBM SPSS (Statistical Package for the Social Sciences) 23, utilizando um nível de significância de 0,05 nos testes estatísticos efetuados. Antes de serem iniciadas as análises, os dados omissos, por não terem ultrapassado 5\% dos casos e, por sua distribuição ser aleatória, foram substituídos pela média (Horton \& Kleinman, 2006).

Para verificar as correlações entre as variáveis foi realizado teste de correlação $\mathrm{r}$ de Pearson, sendo que o coeficiente (r) varia de -1 a 1 . O sinal indica direção positiva ou negativa do relacionamento e o valor sugere a força da relação entre as variáveis, podendo ser interpretados da seguinte maneira: $r=0,10$ até 0,30 (fraco); $r$ $=0,40$ até 0,6 (moderado); $r=0,70$ até 1 (forte).

Para o teste das hipóteses, optou-se pela utilização da análise de regressão hierárquica, que tem como objetivo determinar os melhores preditores (variáveis independentes) de um critério (variável dependente) e/ ou analisar os efeitos mediadores do relacionamento entre as variáveis (Abbad \& Torres, 2002). Para averiguar a significância do poder de mediação das respostas adaptativas na relação entre a adaptabilidade de carreira e empregabilidade percebida foi utilizado o macro PROCESS para SPSS, sendo essa uma ferramenta disponível gratuitamente (http://processmacro.org/).

Assim, para avaliar a significância do poder da mediação foram feitas regressões simultâneas dos efeitos diretos e indiretos conforme procedimento de Preacher e Hayes (2004). O resultado do procedimento mostra, ao final, dois valores no intervalo, que são os limites inferior e superior. Para que o efeito da mediação seja significativo, não pode haver troca de sinal entre esses dois limites, ou seja, se um dos limites for negativo e outro positivo o efeito é nulo ou não significativo. Quando os dois valores dos limites de confiança forem negativos, o efeito indireto é considerado significativo e negativo e, consequentemente, se estes dois valores forem positivos o efeito é considerado significativo e positivo (Hayes, 2013).

\section{Resultados}

Utilizando o Modelo da Adaptação de Carreira como estrutura teórica, esse estudo teve como objetivo, testar a hipótese de que, no processo de transição para o trabalho, a adaptabilidade de carreira prediz a percepção de empregabilidade. Adicionalmente, esperou-se que respostas adaptativas assumissem um poder de mediação na relação entre a adaptabilidade de carreira e a empregabilidade percebida. Os resultados foram apresentados em duas partes, primeiro o estudo descritivo e correlacional, em que foi demonstrado as relações entre as variáveis estudadas, posteriormente o teste das hipóteses.

\section{Estudo Descritivo e Correlacional}

$\mathrm{Na}$ Tabela 1 são apresentadas as médias, os desviospadrão, o valor mínimo e máximo e a consistência interna entre as variáveis do estudo. A amplitude dos coeficientes de precisão varia entre 0,65 e 0,95, o que evidencia índices moderados a elevados de consistência interna.

Em relação à Adaptabilidade de Carreira, a média geral foi de 3,92 (DP $=0,60)$ e as maiores médias foram constatadas nas dimensões Confiança $(M=4,08$ - $D P=0,65)$ e Controle $(M=3,99-D P=0,68)$. Para as Respostas Adaptativas a média geral foi de 3,84 $(D P=0,55)$ e as maiores médias foram nas dimensões Identidade de Carreira $(M=4,04-D P=0,89)$ e Exploração Ampliada $(M=3,82-D P=0,75)$. Por último, a média geral na escala de Empregabilidade foi de 3,44 $(D P=0,50)$, sendo que a média na dimensão Interna $(M=3,68-D P=0,51)$ foi mais elevada que a Externa $(M=3,29-D P=0,62)$.

De um modo geral, como apresentado na Tabela 2 , todas as correlações obtidas mostraram-se estatisticamente significativas, revelando-se assim uma relação 
Tabela 1.

Valores Médios, Desvio Padrão, Curtose, Assimetria, Valores Mínimo e Máximo e Consistência Interna (N=387)

\begin{tabular}{|c|c|c|c|c|c|c|c|}
\hline & & M & $\mathrm{DP}$ & Curt & Assi & Min/Max & $\alpha$ \\
\hline 1 & Adaptabilidade Carreira & 3.92 & .60 & -.353 & -.323 & $2 / 5$ & .95 \\
\hline 2 & Preocupação & 3.80 & .76 & -.498 & -.386 & $1 / 5$ & .90 \\
\hline 3 & Controle & 3.99 & .68 & -.165 & -.501 & $1 / 5$ & .84 \\
\hline 4 & Curiosidade & 3.80 & .74 & -.365 & -.366 & $1 / 5$ & .88 \\
\hline 5 & Confiança & 4.08 & .65 & -.032 & -.539 & $2 / 5$ & .89 \\
\hline 6 & Respostas Adaptativas & 3.83 & .60 & .364 & -.574 & $1 / 5$ & .91 \\
\hline 7 & Identidade Carreira & 4.04 & .89 & .264 & -.947 & $1 / 5$ & .84 \\
\hline 8 & Decisão Carreira & 3.70 & .89 & -.266 & -.440 & $1 / 5$ & .86 \\
\hline 9 & Autoeficácia Profissional & 3.76 & .68 & -.224 & -.257 & $1 / 5$ & .84 \\
\hline 10 & Exploração Ampliada & 3.82 & .75 & .437 & -.596 & $1 / 5$ & .80 \\
\hline 11 & Empregabilidade & 3.44 & .50 & .365 & -.265 & $1 / 5$ & .82 \\
\hline 12 & Interna & 3.68 & .51 & .750 & -.327 & $1 / 5$ & .65 \\
\hline 13 & Externa & 3.29 & .62 & .018 & -.301 & $1 / 5$ & .80 \\
\hline
\end{tabular}

$* * \mathrm{p}<0.01$.

Tabela 2.

Correlações entre as Variáveis em Estudo $(N=387)$

\begin{tabular}{|c|c|c|c|c|c|c|c|c|c|c|c|c|c|c|}
\hline & & 1 & 2 & 3 & 4 & 5 & 6 & 7 & 8 & 9 & 10 & 11 & 12 & 13 \\
\hline 1 & Adaptabilidade Carreira & 1 & & & & & & & & & & & & \\
\hline 2 & Preocupação & $834^{* *}$ & 1 & & & & & & & & & & & \\
\hline 3 & Controle & $847^{* *}$ &, $625^{* *}$ & 1 & & & & & & & & & & \\
\hline 4 & Curiosidade &, $865^{* *}$ &, $596^{* *}$ &, $633^{* *}$ & 1 & & & & & & & & & \\
\hline 5 & Confiança &, $850^{* *}$ &, $584^{* *}$ &, $632^{* *}$ &, $699^{* *}$ & 1 & & & & & & & & \\
\hline 6 & Respostas Adaptativas &, $603^{* *}$ & $623^{* *}$ &, $500^{* *}$ &, $446^{* *}$ &, $470^{* *}$ & 1 & & & & & & & \\
\hline 7 & Identidade Carreira &, $309^{* *}$ & $348^{* *}$ & $288^{* *}$ &, $188^{* *}$ & $2222^{* *}$ &, $727^{* *}$ & 1 & & & & & & \\
\hline 8 & Decisão Carreira &, $534^{* *}$ & $613^{* *}$ &, $436^{* *}$ &, $358^{* *}$ &, $395^{* *}$ &, $795^{* *}$ & ,474 & 1 & & & & & \\
\hline 9 & Autoeficácia Profissional &, $527^{* *}$ &, $481^{* *}$ &, $452^{* *}$ &, $424^{* *}$ &, $433^{* *}$ & $834^{* *}$ &, $433^{* *}$ &, $564^{* *}$ & 1 & & & & \\
\hline 10 & Exploração Ampliada &, $485^{* *}$ &, $493^{* *}$ &, $352^{* *}$ &, $400^{* *}$ & $391^{* *}$ &, $702^{* *}$ & $296^{* *}$ &, $453^{* *}$ &, $473^{* *}$ & 1 & & & \\
\hline 11 & Empregabilidade &, $425^{* *}$ &, $440^{* *}$ &, $332^{* *}$ &, $322^{* *}$ & $343^{* *}$ &, $465^{* *}$ & $390^{* *}$ & $376^{* *}$ &, $340^{* *}$ &, $325^{* *}$ & 1 & & \\
\hline 12 & Interna &, $520^{* *}$ &, $493^{* *}$ &, $466^{* *}$ &, $382^{* *}$ &, $426^{* *}$ &, $559^{* *}$ &, $374^{* *}$ &, $438^{* *}$ &, $518^{* *}$ &, $364^{* *}$ &, $721^{* *}$ & 1 & \\
\hline 13 & Externa & $294^{* *}$ & $327^{* *}$ & $200^{* *}$ &, $229^{* *}$ & $234^{* *}$ &, $326^{* *}$ &, $320^{* *}$ &, $271^{* *}$ &, $184^{* *}$ & $241^{* *}$ &, $939^{* *}$ &, $440^{* *}$ & 1 \\
\hline
\end{tabular}

$* * \mathrm{p}<0.01$.

direta entre as dimensões dos três instrumentos de medida. Além disso, todas as dimensões da escala de Adaptabilidade de Carreira apresentam correlações positivas e de intensidade moderada com as Respostas
Adaptativas $(\mathrm{r}=0,56-\mathrm{p}<0,01)$ e com a Empregabilidade Percebida $(\mathrm{r}=0,42-\mathrm{p}<0,01)$. Os valores mais notórios de correlação entre as dimensões da Adaptabilidade de Carreira e as demais variáveis foram encontrados 
entre a Preocupação e a Decisão de Carreira ( $\mathrm{r}=0,61$ - $\mathrm{p}<0,01)$ e entre a Preocupação e as Respostas Adaptativas $(\mathrm{r}=0,58-\mathrm{p}<0,01)$.

Em relação às Respostas Adaptativas, sobressai-se a correlação entre a Autoeficácia Profissional e a Decisão de Carreira $(r=0,56-p<0,01)$ e Decisão de Carreira e Identidade de Carreira $(r=0,47-p<0,01)$. Dando ênfase às correlações Empregabilidade Percebida, a correlação entre a dimensão interna e externa foi positiva de intensidade moderada $(\mathrm{r}=0,44-\mathrm{p}<0,01)$. Ademais, as medidas globais de cada uma das escalas enfocadas nesse estudo correlacionam de forma positiva, direta e de intensidade moderada, com a amplitude das relações situada entre 0,42 e $0,61(p<0,01)$.

\section{Teste das Hipóteses: Estudos de Predição e Mediação}

As hipóteses deste estudo basearam-se nas premissas do Modelo da Adaptação de Carreira. Em um primeiro momento buscou-se avaliar o efeito preditor da adaptabilidade de carreira em relação à percepção de empregabilidade. Em seguida, incluiu-se no modelo as respostas adaptativas como variável mediadora, como mostra a Figura 2.

De acordo com a Figura 2, o efeito direto representado pela trajetória entre a adaptabilidade de carreira e a empregabilidade percebida, sem as respostas adaptativas, foi significativa com valor de 0,3579 ( $p<0,01)$. Com a inclusão da variável mediadora no modelo, o efeito indireto representado pela trajetória entre adaptabilidade de carreira e as respostas adaptativas foi de $0,4588(\mathrm{p}<0,01)$ e o valor da trajetória entre as respostas adaptativas e empregabilidade percebida foi de 0,2548 $(\mathrm{p}<0,01)$, ambas com valores significativos. Além disso, é importante notar que quando as respostas adaptativas foram adicionadas na equação, o efeito direto da adaptabilidade de carreira na empregabilidade percebida diminuiu. Estes resultados indicam um possível efeito de mediação realizada pelas respostas adaptativas. Contudo, essa mediação foi parcial, uma vez que com a inclusão da variável mediadora o poder preditivo da adaptabilidade de carreira sobre a empregabilidade percebida diminuiu, ou seja, continuou significativo.

Para confirmar a significância do poder mediador das respostas adaptativas na relação entre adaptabilidade de carreira e empregabilidade percebida, recorreu-se aos valores dos intervalos de confiança do efeito indireto (Tabela 3). Os valores dos intervalos de confiança do efeito indireto se mantiveram na mesma direção, indicando significância do poder mediador das respostas adaptativas.

Por fim, para melhor compreender como as diferentes dimensões da adaptabilidade de carreira e das respostas adaptativas predizem a empregabilidade percebida empregou-se a análise de regressão hierárquica múltipla. Relativamente as variáveis independentes, foi incluído um primeiro bloco (Bloco I) com as subescalas

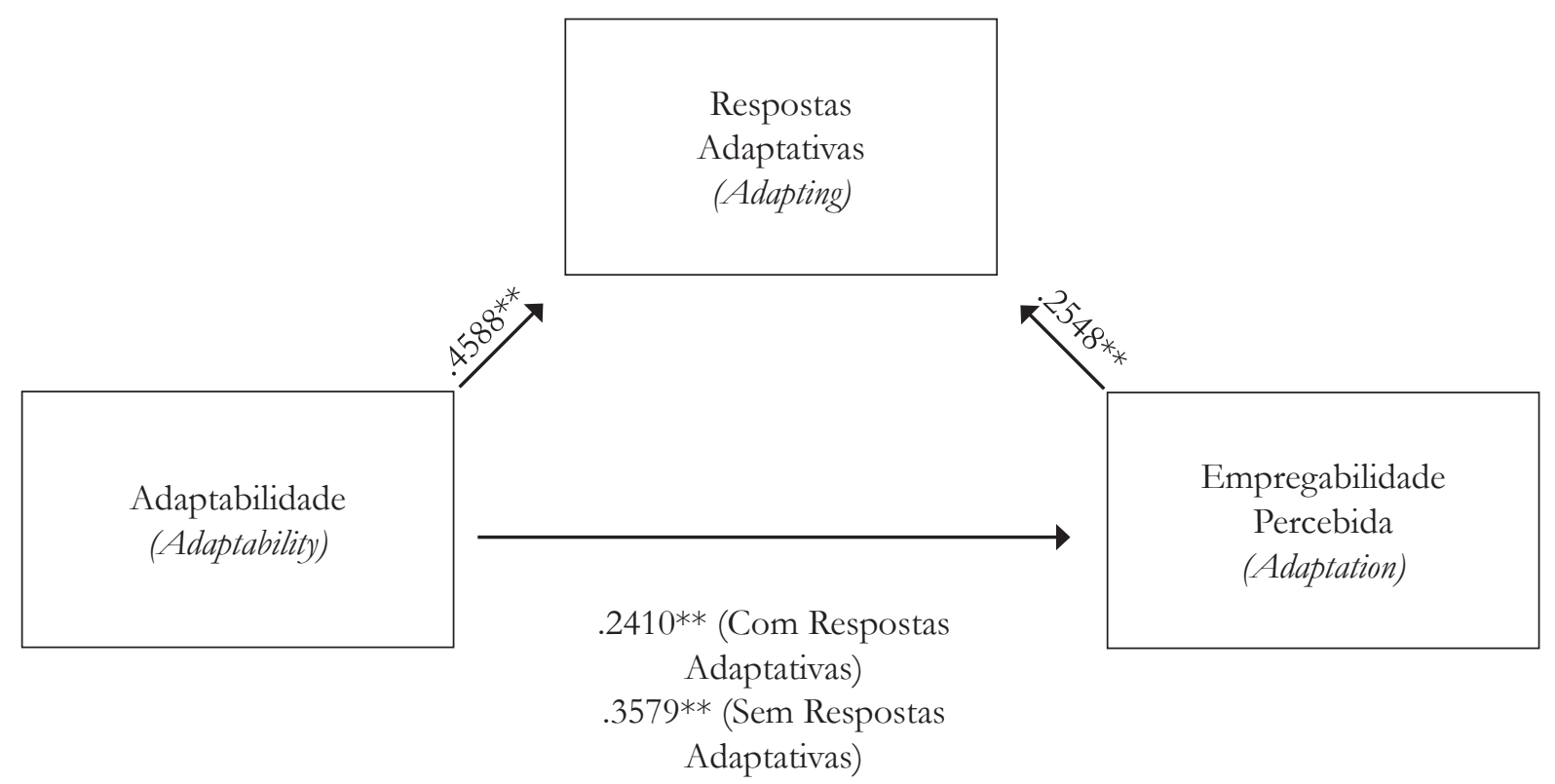

Figura 2. Modelo de Mediação entre Adaptabilidade de Carreira e a Empregabilidade Percebida (H1) 
da adaptabilidade de carreira e um segundo bloco (Bloco II) com as subescalas da adaptabilidade de carreira em conjunto com as respostas adaptativas. A Tabela 4 mostra que a empregabilidade percebida e suas diferentes subescalas foram consideradas variáveis dependentes.

Em relação à empregabilidade global, o primeiro bloco explica cerca de $19,8 \%$ da variância $(F=24,81$ $p<0,01)$, sendo a subescala da preocupação $(\beta=0,21$ - $t=5,46-p<0,01)$ o único preditor individual significativo. Com a introdução do bloco II verificou-se mudanças significativas na predição aumentando o valor da variância explicada para $25,9 \%(F=17,90-p<0,01)$, sendo as dimensões da preocupação $(\beta=0,21-t=3,11$ $-p<0,01)$ e da identidade de carreira $(\beta=0,23-t=4,65$ $-p<0,01)$ os preditores individuais significativos.
Considerando a empregabilidade interna, a variância explicada pelo bloco I $(F=39,52-p<0,01)$ é de $28 \%$ apresentando como preditores individuais as dimensões da preocupação $(\beta=0,29-t=4,98-p<0,01)$, do controle $(\beta=0,20-t=3,31-p<0,01)$. O bloco II $(F=29,28-p<0,01)$, por sua vez, incrementa o valor da variância explicada para $37 \%$, sendo as dimensões da preocupação $(\beta=0,16-t=2,53-p<0,01)$, do controle $(\beta=0,14-t=2,44-p<0,01)$, da identidade de carreira $(\beta=0,12-t=2,64-p<0,01)$ e da autoeficácia ocupacional $(\beta=0,25-t=4,77-p<0,01)$ os preditores individuais significativos.

Em relação à empregabilidade externa o bloco I $(F=11,96-p<0,01)$, explica cerca de $10,2 \%$ da variância, sendo a dimensão da preocupação $(\beta=0,20-t=4,48$ $-p<0,01)$ o único preditor individual significativo. Com

Tabela 3.

Efeito mediador das Respostas Adaptativas na relação entre a Adaptabilidade de Carreira e Empregabilidade Percebida

\begin{tabular}{lcccccc}
\hline & Efeito & Erro & T & Sig & IC Inferior & IC Superior \\
\hline Efeito Total & .3579 & .0388 & 9.2154 &, 0000 & .2815 & .4343 \\
Efeito Direto & .2410 & .0438 & 5.4971 & .0000 & .1548 & .3272 \\
Efeito Indireto (dependência) & .1169 & .0277 & & & .0640 & .1743 \\
\hline
\end{tabular}

Tabela 4.

Regressões Hierárquicas para a Empregabilidade Percebida. $(N=387)$

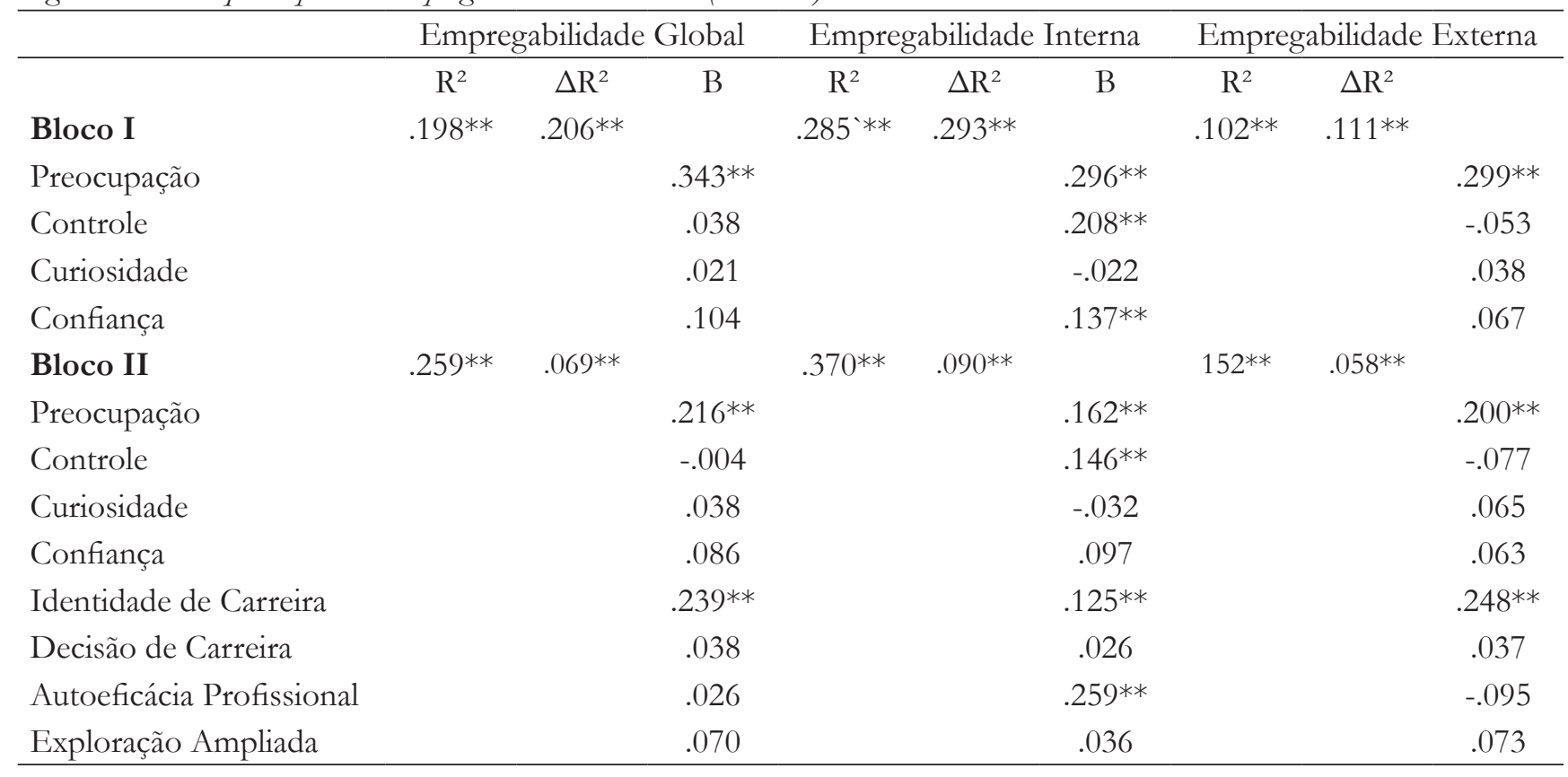

** $\mathrm{p}<0.01$. 
a introdução do bloco II $(F=9,62-p<0,01)$ este valor aumenta para $15,2 \%$, sendo as dimensões da preocupação $(\beta=0,20-t=2,69-p<0,01)$ e da identidade de carreira $(\beta=0,24-t=4,51-p<0,01)$ os únicos preditores individuais significativos.

\section{Discussão e Considerações Finais}

Em um primeiro momento, tal como esperado, as análises de correlações sugerem que a Escala de Adaptabilidade de Carreira relaciona-se de forma significativa e positiva com todas as suas dimensões: Preocupação, Controle, Curiosidade e Confiança. Este resultado vai ao encontro com outros estudos (Savickas \& Porfeli, 2012; Duarte et al., 2012; Gamboa et al., 2014), podendo ainda ser considerados como indicadores da validade do instrumento.

É possível verificar ainda associações positivas e moderadas entre adaptabilidade de carreira e a empregabilidade percebida, principalmente no que diz respeito à dimensão interna da empregabilidade. Estes resultados podem ser analisados levando em consideração a definição de empregabilidade, sendo esta entendida como uma forma de adaptação ativa, que facilita a identificação e a assimilação de oportunidades de carreira, por parte dos indivíduos (Fugate, Kinicki \& Ashforth 2004; Fugate \& Kinicki, 2008). Em conversa com o estudo de Hirschi et. al. (2015), os resultados correlacionais aqui relatados sugerem que a adaptabilidade de carreira, as respostas adaptativas e a empregabilidade percebida avaliam constructos teoricamente e empiricamente diferentes.

Em relação às respostas adaptativas, os resultados corroboram os achados de Hirschi et al. (2015) e Rudolph et. al. (2017), uma vez que sugerem uma relação positiva e moderada entre a adaptabilidade de carreira e as dimensões das respostas adaptativas. Nesse sentido, os recursos da adaptabilidade de carreira parecem estar relacionados às respostas adaptativas em diferentes condições.

No teste das hipóteses, os resultados da relação entre a adaptabilidade de carreira e a empregabilidade percebida confirmaram as expectativas teóricas, uma vez que foi observado um efeito direto e significativo da adaptabilidade de carreira sobre a empregabilidade percebida. Em um segundo momento, confirmou-se o poder mediador das respostas adaptativas, pois se observou queda dos valores da trajetória direta (adaptabilidade de carreira -> empregabilidade percebida) quando as respostas adaptativas foram incluídas na equação. Apesar da diminuição dos valores, a trajetória continuou sendo significativa, o que sugeriu um efeito mediador parcial das respostas adaptativas em relação à empregabilidade percebida.

Em conjunto, os resultados encontrados neste estudo, com uma amostra de estudantes concluintes do ensino superior, sugerem que a adaptabilidade de carreira é uma variável antecedente da empregabilidade percebida, sendo esta relação parcialmente mediada pelas respostas adaptativas. Estes resultados reforçam que a investigação dos mediadores pode ser frutífera para enriquecer a compreensão de caminhos através dos quais a adaptabilidade de carreira se manifesta em respostas adaptativas e resultados no domínio da carreira.

Analisando o poder preditivo das dimensões da adaptabilidade de carreira e das respostas adaptativas, tem-se que em conjunto, explicam aproximadamente, $26 \%$ da percepção de empregabilidade geral. Assim como no estudo de Hirschi et. al. (2015), em relação à adaptabilidade de carreira, a dimensão da preocupação surge como o único preditor individual significativo de todas as dimensões da empregabilidade percebida na transição para o trabalho. Isto implica que nem todas as quatro dimensões da adaptabilidade de carreira são igualmente fortes preditores, como já indicado por pesquisas anteriores (Zacher, 2014). Isso sugere que os pesquisadores devem tratar a adaptabilidade de carreira como uma construção multidimensional cujos componentes não são mutuamente intercambiáveis. E, portanto, merecem uma investigação dos efeitos das diferentes dimensões de adaptabilidade de carreira em relação aos resultados da adaptação (Hirschi et. al., 2015).

Em relação às respostas adaptativas, a identidade de carreira aparece, individualmente, como o único preditor significativo de todas as dimensões da empregabilidade percebida. Isso corrobora os estudos de Lo Presti e Pluviano (2016), que propõe que a identidade de carreira é um componente importante de empregabilidade, em que indivíduos que são capazes de identificar e apreciar o alcance das suas experiências de trabalho, harmonizando com os seus objetivos profissionais futuros.

Mais precisamente, podemos afirmar que o constructo adaptabilidade de carreira remete para competências mais gerais, relacionadas à capacidade de antecipar mudanças e implementar respostas adaptativas e flexíveis, (Savickas, 2005, 2013; Savickas et al., 2009), as respostas adaptativas refere-se ao desempenho 
de comportamentos adaptativos necessários no processo de construção da carreira (Hirschi et al., 2015), enquanto a empregabilidade percebida surge associada a competências mais especificas de procura e manutenção do emprego, decorrentes da organização individual de experiências mais concretas (Guzman \& Choi, 2013; Rothwell et al., 2008).

No que se refere às contribuições deste estudo em termos da investigação, infere-se que a sua relevância se dá a partir da atualidade das pesquisas sobre a adaptabilidade de carreira e o Modelo da Adaptação de Carreira (Hirschi et al., 2015; Rudolph et al., 2017). Além disso, é necessário ressaltar que a literatura sobre carreira aponta a transição universidade-trabalho como uma das principais transições que os jovens enfrentarão, uma vez que traz consequências significativas para a consolidação de sua identidade pessoal e ocupacional e para a promoção do seu bem-estar (Oliveira, Detomini \& Melo-Silva, 2013). Nesta perspectiva, e por se reconhecer cada vez mais o papel da agência individual na organização do comportamento vocacional, os construtos adaptabilidade de carreira e empregabilidade percebida são apontados como recursos psicossociais centrais para o desenvolvimento e a construção da carreira, sendo particularmente importantes para os estudantes do ensino superior em transição para a vida profissional (Gamboa et al., 2014).

Apesar das contribuições do estudo, em uma amostra de estudantes universitários concluintes do ensino superior e em transição para o trabalho, este aspecto pode ser também considerado uma limitação do estudo. Embora esta amostra seja adequada para investigar os objetivos propostos, permitindo comparações significativas com outros estudos sobre a mesma temática, isso implica que os resultados não podem, necessariamente, ser generalizados e aplicados a uma população trabalhadora. Nesse sentido, pesquisas futuras devem tentar replicar os resultados com amostras diferentes para fornecer um conhecimento mais generalizável de como as diferentes facetas da adaptabilidade de carreira se relacionam entre si.

Em suma, é necessário ressaltar também que o impacto da transição universidade-trabalho tem assumido uma relevância cada vez maior nas pesquisas conteporâneas. Nesse sentido, é de grande importância que as instituições de ensino superior proporcionem experiências que contribuam para o desenvolvimento de recursos psicológicos relacionados à adaptabilidade de carreira nas suas diferentes dimensões, facilitando essa transição, ressaltando assim a importância da adaptabilidade de carreira e das respostas adaptativas para o fortalecimento da empregabilidade percebida dos estudantes universitários no processo de transição para o trabalho.

\section{Referências}

Abbad, G. S., Torres, C. V. (2002). Regressão múltipla stepwise e hierárquica em psicologia organizacional: Aplicações, problemas e soluções. Estudos de Psicologia, 7, 19- 29.

Ambiel, R. A. M., Carvalho, L. F., Martins, G. H. M., Tofoli, L. (2016). Comparing the adaptabilities of Brazilian adolescent students and adult workers. Journal of Vocational Behavior, 94, 20-27. doi: 10.1016/j.jvb.2016.02.005

Ambiel, R. A., Hernández, D. N., \& Martins, G. H. (2016). Relações entre adaptabilidade de carreira e vivências acadêmicas no ensino superior. Psicologia desde el Caribe, 33, 159-168. doi: 10.14482/ psdc.33.2.7071

Audibert, A., \& Teixeira, M. A. P. (2015). Escala de Adaptabilidade de Carreira: Evidências de Validade em Universitários Brasileiros. Revista Brasileira de Orientação Profissional, 16(1), 83-93.

Birnbaum, M. H. (2004). Human research and data collection via the Internet. Annual Review of Psychologist, 55, 803-832. doi: 10.12691/rpbs-4-1-2

Brown, S. D., \& Lent, R. W. (2016). Vocational psychology: Agency, equity, and well-being. Annual Review of Psychology, 67, 541-565.

Carvalho, L. F., Moreira, T. C., Ambiel, R. A. M. (2017). Relações entre adaptabilidade de carreira e traços patológicos da personalidade em trabalhadores brasileiros. Revista Psicologia Organizações e Trabalho, 17(3), 159-164. doi: 10.17652/rpot/2017.3.12931

Duarte M. E., Soares M. C., Fraga S., Rafael M., Lima M. R, Paredes, I Agostinho, R., Djaló, A. (2012). Career Adapt-Abilities Scale - Portugal Form: Psychometric Properties and Relationships to Employment Status. Journal of Vocational Behavior. 80, 725-729. doi: 10.1016/j.jvb.2012.01.019,

Fraga, S. (2012). Adaptabilidade e Empregabilidade numa Perspetiva Construtivista: Estudo com desempregados em contexto de formação. Tese de Doutoramento em Psicologia (Psicologia dos Recursos Humanos, do 
Trabalho e das Organizações). Faculdade de Psicologia da Universidade de Lisboa, Lisboa.

Fugate, M., Kinicki, A. J., \& Ashforth, B. E. (2004). Employability: A psycho-social construct, its dimensions, and applications. Journal of Vocational Behaviour, 65, 14-38. doi: 10.1016/j.jvb.2003.10.005

Fugate, M., \& Kinicki, A. J. (2008). A dispositional approach to employability: Development of a measure and test of implications for employee reactions to organizational change. Journal of Occupational and Organizational Psychology, 81(3), 503-527. doi: 10.1348/096317907X241579

Gamboa, V., Paixão, O., Palma, A. I. (2014). Adaptabilidade de Carreira e Autoeficácia na Transição para o Trabalho: O papel da Empregabilidade Percebida - Estudo com Estudantes do Ensino Superior. Revista Portuguesa de Pedagogia 48(2). 133-156. doi: 10.1016/j.jvb.2012.01.019,

Gosling, S. D., Vazire, S., Srivastasa., \& Johnm O. (2004). Should we trust web-based studies? A comparative analyses of six preconceptions about Internet questionnaires. American Psychologist, 59, 390-440.

Guilbert, L., Bernaud, J. L., Gouvernet, B., Rossier, J. (2015). Employability: review and research prospects. International Journal for Education and Vocational Guidance. 16(1). 69-89. doi: 10.1007/ s10775-015-9288-4

Guzman, A. \& Choi, K. (2013) The relations of employability skills to career adaptability among technical school students. Journal of Vocational Behavior, 82, 199-207.

Hayes, A. F. (2013). Model templates for SPSS and SAS. Behavior Research Methods, Instruments, \& Computers, 36, 717-731.

Hirschi, A., Herrmann, A., \& Keller, A. (2015). Career adaptivity, adaptability, and adapting: A conceptual and empirical investigation. Journal of Vocational Behavior, 87, 1-10. doi: 10.1016/j.jvb.2014.11.008

Horton, N. J., \& Kleinman, K. P. (2006) Much Ado About Nothing: A comparison of missing data methods and software to fit incomplete data regression models. The American Statistician, 61, 79-90. doi: 10.1198/000313007X172556

Lo Presti, A., \& Pluviano, S. (2016). Looking for a route in turbulent waters: Employability as a compass for career success. Organizational Psychology Review, 6(2) 192-211. doi: 10.1177/2041386615589398

McArdle, S., Waters, L., Briscoe, J. P., \& Hall, D. T. (2007). Employability during unemployment: Adaptability, career identity and human and social capital. Journal of Vocational Behavior, 71(2), 247264. doi: 10.1016/j.jvb.2007.06.003

McMahon, M., Watson, M., \& Bimrose, J. (2012). Career adaptability: A qualitative understanding from the stories of older women. Journal of Vocational Behavior, 80, 762-768. doi: 10.1016/j.jvb.2012.01.016

Oliveira, C. M., Detomini, V. C., Melo-Silva, L. L. (2013) Sucessonatransiçãouniversidade-trabalho:expectativas deuniversitários formandos. Psicologiaem Revista, 19(3). doi:10.5752/P.1678-9563.2013v19n3p497

Preacher, K. J., Hayes, A. F. (2004). SPSS and SAS procedures for estimating indirect effects in simple mediation models. Behavior Research Methods, Instruments, and Computers, 36, 717-731. doi:10.3758/ BF03206553.

Rothwell, A., Herbert, I., Rothwell F. (2008). Selfperceived employability: Construction and initial validation of a scale for university students. Journal of Vocational Behavior. 73(1), 1-12. doi: 10.1016/j. jvb.2009.05.002

Rothwell, A., Jewell, S., \& Hardie, M. (2009). Selfperceived employability: Investigating the responses of postgraduate students. Journal of Vocational Behavior, 75(1), 152161. doi: 10.1016/j.jvb.2009.05.002

Rudolph, C. W., Lavigne, K. N., Zacher, H. (2017). Career adaptability: A meta-analysis of relationships with measures of adaptivity, adapting responses, and adaptation result. Journal of Vocational Behavior, 98(1), 17-34. doi: 10.1016/j.jvb.2017.05.008

Savickas, M. L. (2013). Career construction theory and practice. In S. Brown \& R. Lent (Eds.), Career development and counselling. Putting theory and research to work (pp. 147-183). New Jersey: John Wiley \& Sons.

Savickas, M. L. (2005). The theory and practice of career construction. In R. W. Lent, \& S. D. Brown (eds.), Career development and counseling: putting theory and research to work (pp. 42-70). Hoboken, New Jersey: John Wiley \& Sons.

Savickas, M. L., \& Porfeli, E.J. (2012). Career AdaptAbilities Scale: Construction, reliability, and 
measurement equivalence across 13 countries. Journal of Vocational Behavior, 80, 661-673. doi: 10.1016/j.jvb.2012.01.012

Savickas, M., Nota, L., Rossier, J., Dauwalder, J-P., Duarte, M. E., Guichard, J., Soresi, S., Van Esbroeck, R., \& Van Vianen, A. (2009). Life designing: A paradigm for career construction in the 21 st Century. Journal of Vocational Behavior, 75(3), 239-250. doi: 10.1016/j.jvb.2009.04.004

Teixeira, M. A. P., Bardagi, M. P., Lassance, M. C. P., Magalhães, M. O., Duarte, M. E. (2012). Career Adapt-Abilities Scale-Brazilian Form: Psychometric properties and relationships to personality. Journal of Vocational Behavior 80. 680-685. doi: 10.1016/j.jvb.2012.01.007

Teixeira, M. A. P., Oliveira, M. C. D., Melo-Silva, L. L., \& Taveira, M. D. C. (2019). Escalas de Desenvolvimento de Carreira de Universitários: construção, características psicométricas e modelo das respostas adaptativas. Revista Psicologia Organizaçōes e Trabalho, 19(3), 703-712. doi: 10.17652/ rpot/2019.3.16557

Teixeira, M. A. P., Oliveira, M. C., Melo-Silva, L. L., Taveira, M. C. Escalas de Desenvolvimento de Carreira e Modelo das Respostas Adaptativas para Universitários. Submetido à publicação.

Van der Heijde, C. M. (2014). Employability and selfregulation in contemporary careers. In M. Coetzee (Ed.), Psychosocial career meta-capacities: dynamics of contemporary career development (pp. 7-18). Dordrecht, The Netherlands: Springer International. doi: 10.1007/978-3-319-00645-1_1

Recebido em: 30/06/2018

Reformulado em: $17 / 10 / 2018$

Aprovado em: 24/10/2018 
Sobre as autoras:

Mariana Rita Machado Ladeira é psicóloga, graduada em Psicologia pela Universidade Federal do Triângulo Mineiro (UFTM), participante do Grupo de Pesquisa em Educação e Construção da Carreira (GPECC/UFTM). ORCID: https://orcid.org/0000-0002-4035-2639

E-mail:marianarladeira@gmail.com

Marina Cardoso de Oliveira é psicóloga, doutora em Psicologia pela Faculdade de Filosofia, Ciências e Letras de Ribeirão Preto (FFCLRP-USP). Professora Adjunta do Departamento de Psicologia da Universidade Federal do Triângulo Mineiro (UFTM). Coordenadora do Laboratório e do Grupo de Pesquisa em Educação e Construção da Carreira (LaPECC/GPECC).

ORCID: https://orcid.org/0000-0002-8715-2853

E-mail:mco.uftm@gmail.com

Lucy Leal Melo-Silva é doutora em Psicologia, docente sênior do Departamento de Psicologia da Faculdade de Filosofia, Ciências e Letras de Ribeirão Preto (FFCLRP-USP). Orienta na Pós-Graduação no domínio da Orientação Profissional e de Carreira. Coordenadora do Carreira Lab. Coeditora da RBOP.

ORCID: https://orcid.org/0000-0002-5890-9896

E-mail: lucileal@ffclrp.usp.br

Maria do Céu Taveira é doutora em Psicologia da Educação, docente da Escola de Psicologia da Universidade do Minho, em Portugal. Coordena o grupo de investigação em Desenvolvimento de Carreira e Aconselhamento no Centro de Investigação em Psicologia (CIPsi). É membro fundadora e Presidente da Mesa da Assembleia Geral da Associação Portuguesa para o Desenvolvimento de Carreira.

ORCID: https://orcid.org/0000-0003-1762-8702

E-mail:ceuta@psi.uminho.pt

Contato com as autoras:

Departamento de Psicologia da UFTM

Av. Getúlio Guaritá, 159, Bairro Abadia

Uberaba-MG, Brasil

CEP: 38025-440

Telefones: (34) 3700-6992 